\title{
A RELATIVE COHOMOLOGY FOR ASSOCIATIVE ALGEBRAS
}

\author{
U. SHUKLA
}

Introduction. In this paper we propose a relative cohomology for associative algebras over a commutative ring. A relative cohomology for associative algebras over a field has been given by Hochschild [2]. In [3] the author has given a cohomology for associative algebras over a commutative ring which is a generalisation of Hochschild's cohomology for associative algebras over a field, a description of which can be found in [1]. In order to be able to show that the relative cohomology proposed here is in the same way a true generalisation of Hochschild's relative cohomology, we require a theorem analogous to [3, Theorem 1$]$. We are unable to prove such a theorem.

I am grateful to the referee for his useful suggestions.

1. The tensor product and Hom of graded modules over graded rings. Let $R=\sum_{p \geqq 0} R^{p}$ be a graded ring, $A=\sum_{p \geq 0} A^{p}$ be a graded right $R$-module and $B=\sum_{p \geq 0} B^{p}$ be a graded left $R$-module. Let $F$ be a graded free abelian group generated by the pairs $(a, b)$, where $a \in A^{p}, b \in B^{q}(p \geqq 0, q \geqq 0)$, the pair $(a, b)$ being a homogeneous element of degree $p+q$. Let $G$ be the graded subgroup generated by the homogeneous elements of the form $\left(a+a^{\prime}, b\right)-(a, b)-\left(a^{\prime}, b\right)$, $\left(a, b+b^{\prime}\right)-(a, b)-\left(a, b^{\prime}\right)$, and $(a \lambda, b)-(-1)^{r}(a, \lambda b)$, where $a, a^{\prime} \in A^{p}$; $b, b^{\prime} \in B^{q} ; \lambda \in R^{r}(p \geqq 0, q \geqq 0, r \geqq 0)$. The factor group $F / G$ is a graded abelian group which we call the tensor product of the graded right $R$-module $A$ and the graded left $R$-module $B$ over the graded ring $R$ and denote it by $A \otimes_{R} B$. We denote the image of the element $(a, b)$ of $F$ in $A \otimes_{R} B$ by $a \otimes b$. Then $\left(a+a^{\prime}\right) \otimes b=a \otimes b+a^{\prime} \otimes b, a \otimes\left(b+b^{\prime}\right)$ $=a \otimes b+a \otimes b^{\prime}$, and $a \lambda \otimes b=(-1)^{r} a \otimes \lambda b$.

Suppose now that both $A$ and $B$ are graded left $R$-modules. We say that $f: A \rightarrow B$ is a graded-R-linear map if $f\left(a+a^{\prime}\right)=f(a)+f\left(a^{\prime}\right)$, and $f(\lambda a)=(-1)^{r} \lambda f(a)$, where $a, a^{\prime} \in A$ and $\lambda$ is a homogeneous element of $R$ of degree $r$. The set of all such graded-R-linear maps is an abelian group which we denote by $\operatorname{Hom}_{R}(A, B)$. When $A$ and $B$ are both right $R$-modules, we define $\operatorname{Hom}_{R}(A, B)$ in an analogous fashion.

The tensor product and Hom defined here are different from the usual tensor product and Hom of graded modules over a graded ring inasmuch as the grading of the base ring $R$ is also taken into account. We shall have occasion to use the usual Hom also in the sequel.

Received by the editors November 23, 1962. 
2. The complex $B(U, V)$. By a set-couple [resp. module-couple, algebra-couple] $(P, Q)$ we mean that $P$ is a set [resp. module, algebra] and $Q$ is a subset [resp. submodule, subalgebra] of $P$. By a map [resp. homomorphism] $f:(P, Q) \rightarrow\left(P^{\prime}, Q^{\prime}\right)$ of set-couples [resp. module-couples, algebra-couples] we understand a map [resp. homomorphism ] $f: P \rightarrow P^{\prime}$ such that $f(Q) \subset Q^{\prime}$.

Let $K$ be a commutative ring with identity $1 \neq 0$. By an algebra we shall mean an associative algebra over $K$ and except when mention is made to the contrary an associative algebra will be understood to possess an identity. A homomorphism of algebras will be understood to map the identity (if there exists one) in to the identity.

Let $(\Lambda, \Gamma)$ be an algebra-couple. We construct (cf. [3, p. 172]) a differential graded algebra-couple $(U, V)$, where $U=\sum_{n \geq 0} U_{n}$, $V=\sum_{n \geq 0} V_{n}$, together with a homomorphism of differential graded algebra-couples $\epsilon:(U, V) \rightarrow(\Lambda, \Gamma)$, the differential and grading in $(\Lambda, \Gamma)$ being trivial, having the following properties:

(i) For every $n \geqq 0, U_{n}=K\left(X_{n}\right)$ [resp. $V_{n}=K\left(Y_{n}\right)$ ], the $K$-free module having the set $X_{n}$ [resp. $Y_{n}$ ] as base.

(ii) An associative multiplication is defined in $U$ [resp. $V$ ] by $K$ linear extension of the maps of set-couples

$$
\left(X_{i} \times X_{j}, Y_{i} \times Y_{j}\right) \rightarrow\left(X_{i+j}, Y_{i+j}\right)
$$

for which $\left(x_{i} \cdot x_{j}\right) \cdot x_{k}=x_{i} \cdot\left(x_{j} \cdot x_{k}\right) ; x_{i} \in X_{i}, x_{j} \in X_{j}, x_{k} \in X_{k}$, where $x_{i} \cdot x_{j}$ denotes the image of $\left(x_{i}, x_{j}\right)$ in $X_{i+j}$. In particular, $\left(U_{0}, V_{0}\right)$ is an associative algebra-couple.

(iii) The restriction of the homomorphism $\epsilon:\left(U_{0}, V_{0}\right) \rightarrow(\Lambda, \Gamma)$ to $\left(X_{0}, Y_{0}\right)$ is a bijective map which preserves multiplication.

(iv) For each $n \geqq 1$, there is a homomorphism of $K$-modulecouples $d_{n}:\left(U_{n}, V_{n}\right) \rightarrow\left(U_{n-1}, V_{n-1}\right)$ such that for $n=1$, the restriction of $d_{1}$ to $\left(X_{1}, Y_{1}\right)$ is a bijective map of $\left(X_{1}, Y_{1}\right)$ onto the kernel-couple $\left(N_{0}, L_{0}\right)$ of $\epsilon$ and that for $n \geqq 2$, the restriction of $d_{n}$ to $\left(X_{n}, Y_{n}\right)$ is a bijective map of $\left(X_{n}, Y_{n}\right)$ onto the kernel-couple $\left(N_{n-1}, L_{n-1}\right)$ of $d_{n-1}$. We define $d_{0}=0$.

(v) We have $d_{i+j}\left(x_{i} \cdot x_{j}\right)=\left(d_{i} x_{i}\right) \cdot x_{j}+(-1)^{i} x_{i} \cdot\left(d_{j} x_{j}\right) ; x_{i} \in X_{i}, x_{j} \in X_{j}$ $(i \geqq 0, j \geqq 0)$, which by $K$-linearity gives an analogous relation when $x_{i} \in U_{i}, x_{j} \in U_{j}(i \geqq 0, j \geqq 0)$.

Let

$$
S(U, V)=\sum_{n \geqq-1} S_{n}(U, V)
$$

where

$$
S_{n}(U, V)=U \otimes_{V} \cdots \otimes_{V} U, \quad(n+2) \text { factors, } n \geqq-1 .
$$


If $x=u_{0} \otimes \cdots \otimes u_{n+1} \in S_{n}(U, V), n \geqq-1$, the grading in $S(U, V)$ is given by

$$
\operatorname{deg} x=n+\sum_{i=0}^{n+1} \operatorname{deg} u_{i}
$$

We define a map $\tau: S_{n-1}(U, V) \rightarrow S_{n}(U, V),(n \geqq 0)$ by the relation $\tau(x)=1 \otimes x$, where $x \in S_{n-1}(U, V)$. Then $\tau$ is a graded- $V$-left-linear and $U$-right-linear map. For

$$
\begin{aligned}
\tau(v x) & =1 \otimes v x=(-1)^{\operatorname{deg} v} v \otimes x=(-1)^{\operatorname{deg} v} v(1 \otimes x) \\
& =(-1)^{\operatorname{deg} v} v \tau(x), \\
\tau(x u) & =1 \otimes x u=(1 \otimes x) u=\tau(x) u,
\end{aligned}
$$

where $x \in S_{n-1}(U, V), v$ is a homogeneous element of $V$ and $u \in U$.

We now define two differentials $\partial_{r}: S_{n}(U, V) \rightarrow S_{n}(U, V)$ and $\partial_{s}: S_{n}(U, V) \rightarrow S_{n-1}(U, V)$ with the help of $d$ and $\tau$ exactly as in [3, p. 166] and set $\partial=\partial_{r}+\partial_{s}$. We thus obtain a $K$-linear homogeneous map $\partial: S(U, V) \rightarrow S(U, V)$ of degree -1 such that $\partial^{2}=0$. The explicit formulas for $\partial_{r}$ and $\partial_{s}$ are

$$
\begin{aligned}
& \partial_{r}\left(u_{0} \otimes \cdots \otimes u_{n+1}\right) \\
& =d u_{0} \otimes u_{1} \otimes \cdots \otimes u_{n+1} \\
& +\sum_{i=1}^{n}(-1)^{i+\operatorname{deg} u_{0}+\cdots+\operatorname{deg} u_{i-1}} u_{0} \otimes \cdots \otimes d u_{i} \otimes \cdots u_{n+1} \\
& +(-1)^{n+\operatorname{deg} u_{0}+\cdots+\operatorname{deg} u_{n}} u_{0} \otimes \cdots \otimes u_{n} \otimes d u_{n+1} \text {; } \\
& \partial_{s}\left(u_{0} \otimes \cdots \otimes u_{n+1}\right) \\
& =\sum_{i=0}^{n}(-1)^{i+\operatorname{deg} u_{0}+\cdots+\operatorname{deg} u_{i}} u_{0} \otimes \cdots \otimes u_{i} u_{i+1} \otimes \cdots \otimes u_{n+1}
\end{aligned}
$$

for $n \geqq 0$.

Let

$$
B(U, V)=\sum_{n \geq 0} S_{n}(U, V)=S(U, V) / S_{-1}(U, V) .
$$

Since $S_{-1}(U, V)$ is stable for $\partial, \partial$ induces a differential (again denoted by $\partial)$ in $B(U, V)$.

3. The relative cohomology of an algebra. Let $M$ be a $\Lambda$-bimodule. The homomorphism $\epsilon:(U, V) \rightarrow(\Lambda, \Gamma)$ induces over $M$ the structure of a $U$-bimodule such that

$$
u m=0=m u, \quad m \in M, u \in U, \operatorname{deg} u>0,
$$

and 


$$
(d u) m=0=m(d u), \quad m \in M, u \in U \text { and } \operatorname{deg} u=1 .
$$

Let $U^{*}$ [resp. $V^{*}$ ] be the opposite algebra of $U$ [resp. $V$ ] and let $U^{e}=U \otimes_{K} U^{*}\left[\right.$ resp. $V^{e}=V \otimes_{K} V^{*}$ ] denote the enveloping algebra of $U$ [resp. $V$ ]. Then $M$ can be given the structure of a left $U^{e}$-module. We denote by $\operatorname{Hom}_{U^{e}}(B(U, V), M)$ the direct sum

$$
\sum_{n \geq 0} \operatorname{Hom}_{U^{\bullet}}\left(S_{n}(U, V), M\right) \text {. }
$$

It can be shown as in [3] that $\operatorname{Hom}_{U^{e}}(B(U, V), M)$ is a complex and the differential $\delta$ is given by $\delta f=f \partial$, where $f \in \operatorname{Hom}_{U^{e}}(B(U, V), M)$.

If we write $U^{n}=U \otimes_{V} \cdots \otimes_{V} U$ ( $n$ factors) with $U^{0}=V$, there is a natural isomorphism

$$
\alpha: \operatorname{Hom}_{V^{\bullet}}\left(U^{n}, M\right) \rightarrow \operatorname{Hom}_{U^{\bullet}}\left(S_{n}(U, V), M\right)
$$

given by

$$
(\alpha f)\left(u_{0} \otimes \cdots \otimes u_{n+1}\right)=u_{0} f\left(u_{1} \otimes \cdots \otimes u_{n}\right) u_{n+1},
$$

where $f \in \operatorname{Hom}_{V^{e}}\left(U^{n}, M\right)$ and $u_{0}, \cdots, u_{n+1} \in U$. We now have an isomorphism

$$
\operatorname{Hom}_{U^{\bullet}}(B(U, V), M) \approx \sum_{n \geq 0} \operatorname{Hom}_{V^{\bullet}}\left(U^{n}, M\right) .
$$

If $f \in \operatorname{Hom}_{V^{e}}\left(U^{n}, M\right)$, then $\delta f=g+h$, where $g \in \operatorname{Hom}_{V^{c}}\left(U^{n}, M\right)$ and $h \in \operatorname{Hom}_{V^{e}}\left(U^{n+1}, M\right)$ such that

$$
\begin{aligned}
g\left(u_{1} \otimes\right. & \left.\cdots \otimes u_{n}\right) \\
& =\sum_{i=1}^{n}(-1)^{i+\operatorname{deg} u_{1}+\cdots+\operatorname{deg} u_{i-1}} f\left(u_{1} \otimes \cdots \otimes d u_{i} \otimes \cdots \otimes u_{n}\right), \\
h\left(u_{1} \otimes\right. & \left.\cdots \otimes u_{n+1}\right)=u_{1} f\left(u_{2} \otimes \cdots \otimes u_{n+1}\right) \\
& +\sum_{i=1}^{n}(-1)^{i+\operatorname{deg} u_{1}+\cdots+\operatorname{deg} u_{i}} f\left(u_{1} \otimes \cdots \otimes u_{i} u_{i+1} \otimes \cdots \otimes u_{n+1}\right) \\
& +(-1)^{n+1+\operatorname{deg} u_{1}+\cdots+\operatorname{deg} u_{n+1}} f\left(u_{1} \otimes \cdots \otimes u_{n}\right) u_{n+1} .
\end{aligned}
$$

Definition. The $K$-module $H^{*}\left(\operatorname{Hom}_{U^{e}}(B(U, V), M)\right)$ is called the relative cohomology module of the $K$-algebra-couple $(\Lambda, \Gamma)$ with coefficients in the $\Lambda$-bimodule $M$ and is denoted by $H^{*}(\Lambda, \Gamma, M)$.

4. Interpretations of $H^{0}(\Lambda, \Gamma, M)$ and $H^{1}(\Lambda, \Gamma, M)$. The relative $n$-cochains with coefficients in $M$ for $n=0,1,2,3$ are as follows (cf. $[3$, p. 182]):

(i) A relative 0 -cochain is an element of $\operatorname{Hom}_{V^{e}}(V, M)$ and may be identified with an element $m \in M$ for which $\gamma m=m \gamma$ for all $\gamma \in \Gamma$.

(ii) A relative 1-cochain is an element of $\operatorname{Hom}_{V^{e}}\left(U_{0}, M\right)$ and is determined by a map $\chi: \Lambda \rightarrow M$ such that 


$$
\chi(\gamma \lambda)=\gamma \chi(\lambda), \quad \chi(\lambda \gamma)=\chi(\lambda) \gamma, \quad \lambda \in \Lambda, \gamma \in \Gamma .
$$

(iii) A relative 2 -cochain is an element of degree 2 of $\operatorname{Hom}_{V^{e}}\left(U \otimes_{V} U, M\right)+\operatorname{Hom}_{V^{e}}(U, M)$ and is determined by two maps

$$
\begin{aligned}
& \chi_{1}: \Lambda \times \Lambda \rightarrow M, \\
& \chi_{2}: N_{0} \rightarrow M,
\end{aligned}
$$

such that $\chi_{1}\left(\gamma \lambda_{1}, \lambda_{2}\right)=\gamma \chi_{1}\left(\lambda_{1}, \lambda_{2}\right), \chi_{1}\left(\lambda_{1} \gamma, \lambda_{2}\right)=\chi_{1}\left(\lambda_{1}, \gamma \lambda_{2}\right), \chi_{1}\left(\lambda_{1}, \lambda_{2} \gamma\right)$ $=\chi_{1}\left(\lambda_{1}, \lambda_{2}\right) \gamma, \chi_{2}\left(\gamma n_{0}\right)=\gamma \chi_{2}\left(n_{0}\right), \chi_{2}\left(n_{0} \gamma\right)=\chi_{2}\left(n_{0}\right) \gamma, \chi_{2}\left(l_{0}\right)=0 ; \lambda_{1}, \lambda_{2} \in \Lambda$, $\gamma \in \Gamma, n_{0} \in N_{0}, l_{0} \in L_{0}$.

(iv) A relative 3 -cochain is determined by four maps

$$
\begin{aligned}
& \pi_{1}: \Lambda \times \Lambda \times \Lambda \rightarrow M, \\
& \pi_{2}: \Lambda \times N_{0} \rightarrow M, \\
& \pi_{3}: N_{0} \times \Lambda \rightarrow M, \\
& \pi_{4}: N_{1} \rightarrow M,
\end{aligned}
$$

such that $\pi_{1}\left(\gamma \lambda_{1}, \lambda_{2}, \lambda_{3}\right)=\gamma \pi_{1}\left(\lambda_{1}, \lambda_{2}, \lambda_{3}\right), \pi_{1}\left(\lambda_{1} \gamma, \lambda_{2}, \lambda_{3}\right)=\pi_{1}\left(\lambda_{1}, \gamma \lambda_{2}, \lambda_{3}\right)$, $\pi_{1}\left(\lambda_{1}, \lambda_{2} \gamma, \lambda_{3}\right)=\pi_{1}\left(\lambda_{1}, \lambda_{2}, \gamma \lambda_{3}\right), \pi_{1}\left(\lambda_{1}, \lambda_{2}, \lambda_{3} \gamma\right)=\pi_{1}\left(\lambda_{1}, \lambda_{2}, \lambda_{3}\right) \gamma, \pi_{2}\left(\gamma \lambda, n_{0}\right)$ $=\gamma \pi_{2}\left(\lambda, n_{0}\right), \pi_{2}\left(\lambda \gamma, n_{0}\right)=\pi_{2}\left(\lambda, \gamma n_{0}\right), \pi_{2}\left(\lambda, n_{0} \gamma\right)=\pi_{2}\left(\lambda, n_{0}\right) \gamma, \pi_{2}\left(\lambda, l_{0}\right)$ $=0, \pi_{3}\left(\gamma n_{0}, \lambda\right)=\gamma \pi_{3}\left(n_{0}, \lambda\right), \pi_{3}\left(n_{0} \gamma, \lambda\right)=\pi_{3}\left(n_{0}, \gamma \lambda\right), \pi_{3}\left(n_{0}, \gamma \lambda\right)$ $=\pi_{3}\left(n_{0}, \lambda\right) \gamma, \pi_{3}\left(l_{0}, \lambda\right)=0, \pi_{4}\left(\gamma n_{1}\right)=\gamma \pi_{4}\left(n_{1}\right), \pi_{4}\left(n_{1} \gamma\right)=\pi_{4}\left(n_{1}\right) \gamma, \pi_{4}\left(l_{1}\right)$ $=0 ; \lambda, \lambda_{1}, \lambda_{2}, \lambda_{3} \in \Lambda, \gamma \in \Gamma, n_{0} \in N_{0}, n_{1} \in N_{1}$, and $l_{0}, l_{1} \in L_{1}$.

Similarly, a relative 4 -cochain is determined by eight maps satisfying a number of relations.

The calculations show that $H^{0}(\Lambda, \Gamma, M)$ is isomorphic to the submodule of the $K$-module $M$ consisting of elements $m \in M$ for which $\lambda m=m \lambda$ for every $\lambda \in \Lambda$. Hence $H^{0}(\Lambda, \Gamma, M)$ coincides with $H^{0}(\Lambda, M)$ defined in [3].

We shall call a $K$-homomorphism $f: \Lambda \rightarrow M$ a crossed $\Gamma$-homomorphism of $\Lambda$ into $M$ if (i) $f\left(\lambda_{1} \lambda_{2}\right)=\lambda_{1} f\left(\lambda_{2}\right)+f\left(\lambda_{1}\right) \lambda_{2}$ for $\lambda_{1}, \lambda_{2} \in \Lambda$; (ii) $f(\gamma \lambda)$ $=\gamma f(\lambda), f(\lambda \gamma)=f(\lambda) \gamma$ for $\gamma \in \Gamma, \lambda \in \Lambda$. The condition (ii) is equivalent to $f(\gamma)=0$ for $\gamma \in \Gamma$. We shall call a crossed $\Gamma$-homomorphism $f: \Lambda \rightarrow M$ a principal $\Gamma$-homomorphism if there exists an element $m \in M$ such that $f(\lambda)=\lambda m-m \lambda, \lambda \in \Lambda$. Such an element $m$ must necessarily satisfy the relation $\gamma m=m \gamma$ for every $\gamma \in \Gamma$. The calculations then show that $H^{1}(\Lambda, \Gamma, M)$ is isomorphic to the quotient of the $K$-module of crossed $\Gamma$-homomorphisms of $\Lambda$ into $M$ by the submodule of principal $\Gamma$ homomorphisms of $\Lambda$ into $M$. There is a natural homomorphism

$$
H^{1}(\Lambda, \Gamma, M) \rightarrow H^{1}(\Lambda, M),
$$

where $H^{1}(\Lambda, M)$ is the 1-cohomology module defined in [3]. This homomorphism is actually a monomorphism, since a principal $K$ - 
homomorphism which is also a crossed $\Gamma$-homomorphism is a principal $\Gamma$-homomorphism.

5. Interpretation of $H^{2}(\Lambda, \Gamma, M)$. Let $\beta: E \rightarrow \Lambda$ be an onto homomorphism of algebras with kernel $M$ and let $\rho: \Gamma \rightarrow E$ be a homomorphism of algebras such that (i) $\beta \rho$ is the identity map over $\Gamma$, (ii) $\rho$ can be extended to a map $\bar{\rho}: \Lambda \rightarrow E$ for which $\beta \bar{\rho}$ is the identity map over $\Lambda$ and $\bar{\rho}(\gamma \lambda)=\rho(\gamma) \bar{\rho}(\lambda), \bar{\rho}(\lambda \gamma)=\bar{\rho}(\lambda) \rho(\gamma), \lambda \in \Lambda, \gamma \in \Gamma$. We say that the triple $(E, \beta, \rho)$ is a relative extension of the algebra-couple $(\Lambda, \Gamma)$ with kernel $M$. We note that $M$ is a subalgebra of $E$ not necessarily having an identity. Two relative extensions $\left(E^{\prime}, \beta, \rho\right)$ and $\left(E^{\prime}, \beta^{\prime}, \rho^{\prime}\right)$ of the algebra-couple $(\Lambda, \Gamma)$ with the same kernel $M$ are said to be equivalent if there exists an isomorphism $\phi: E \rightarrow E^{\prime}$ such that $\beta=\beta^{\prime} \phi$ and $\phi \rho=\rho^{\prime}$.

A relative extension $(E, \beta, \rho)$ is said to be special if the product of any two elements of the kernel $M$ is zero. In this case $\beta$ induces a $\Lambda$-bimodule structure over $M$.

TheOREM 1. Let $(\Lambda, \Gamma)$ be an algebra-couple and let $M$ be a $\Lambda$-bimodule. Then there exists a natural one-one correspondence between the relative cohomology module $H^{2}(\Lambda, \Gamma, M)$ and the set of equivalence classes of special relative extensions of the algebra-couple $(\Lambda, \Gamma)$ with kernel $M$ which induce over $M$ the given $\Lambda$-bimodule structure.

The proof is similar to that of $[3$, Theorem 4].

6. Interpretation of $H^{3}(\Lambda, \Gamma, M)$. Let $A$ be an algebra not necessarily having an identity. Let $(E, \beta, \rho)$ be a relative extension of the algebra-couple $(\Lambda, \Gamma)$ with kernel $A$. We do not suppose that the product of any two elements of $A$ is zero. Let $M_{A}$ denote the algebra of bimultiplications of $A$ and let $P_{A}$ denote the quotient algebra of exterior bimultiplications of $A[3, \mathrm{p} .197]$. Since $A$ is a two-sided ideal in $E$, the map which assigns to every element $e$ of $E$ the inner bimultiplication of $E$ induced by $e$ gives a homomorphism of algebras $\nu: E \rightarrow M_{A}$. Since $A$ is mapped into the subalgebra of inner bimultiplications, $\nu$ induces a homomorphism of algebras $\theta: \Lambda \rightarrow P_{A}$. If we compose $\nu$ with the homomorphism $\rho: \Gamma \rightarrow E$, we get a homomorphism of algebras $\sigma: \Gamma \rightarrow M_{A}$. Since $\rho$ can be extended to a map $\bar{\rho}: \Lambda \rightarrow E$ for which $\beta \bar{\rho}=\mathrm{id}_{\Lambda}$, and $\bar{\rho}(\gamma \lambda)=\rho(\gamma) \bar{\rho}(\lambda), \bar{\rho}(\lambda \gamma)=\bar{\rho}(\lambda) \rho(\gamma)$ where $\lambda \in \Lambda$, $\gamma \in \Gamma$, it follows that $\sigma$ can be extended to a map $\bar{\sigma}: \Lambda \rightarrow M_{A}$ for which $\zeta \bar{\sigma}=\theta$, and $\bar{\sigma}(\gamma \lambda)=\sigma(\gamma) \bar{\sigma}(\lambda), \bar{\sigma}(\lambda \gamma)=\bar{\sigma}(\lambda) \sigma(\gamma)$ where $\lambda \in \Lambda, \gamma \in \Gamma, \zeta$ being the natural homomorphism of $M_{A}$ onto $P_{A}$. We see that a relative extension determines two homomorphisms of algebras $\theta$ and $\sigma$ which satisfy the properties just described. Both $\theta$ and $\sigma$ are regular homomorphisms [3, p. 199]. 
Conversely, suppose we are given two regular homomorphisms $\theta: \Lambda \rightarrow P_{A}$ and $\sigma: \Gamma \rightarrow M_{A}$ which are such that it is possible to define a map $\bar{\sigma}: \Lambda \rightarrow M_{A}$ having the following properties: (i) $\zeta \bar{\sigma}=\theta$, (ii) the restriction of the map $\bar{\sigma}: \Lambda \rightarrow M_{A}$ to $\Gamma$ is the given homomorphism $\sigma: \Gamma \rightarrow M_{A}$, and (iii) $\bar{\sigma}(\gamma \lambda)=\sigma(\gamma) \bar{\sigma}(\lambda), \bar{\sigma}(\lambda \gamma)=\bar{\sigma}(\lambda) \sigma(\gamma)$ where $\lambda \in \Lambda$, $\gamma \in \Gamma$. Does there exist a relative extension $(E, \beta, \rho)$ of the algebracouple $(\Lambda, \Gamma)$ with kernel $A$ which determines the two regular homomorphisms $\theta$ and $\sigma$ ? We shall associate with the pair $(\theta, \sigma)$ an element of the relative cohomology module $H^{3}\left(\Lambda, \Gamma, C_{A}\right)$, where $C_{A}$ is the bicentre [3, p. 198] of $A$. We shall call this element the obstruction of $(\theta, \sigma)$ and shall denote it by $\xi_{(\theta, \sigma)}$.

Choose a map $\bar{\sigma}: \Lambda \rightarrow M_{A}$ having the properties given above. Then $\bar{\sigma}(0)=0$ and $\bar{\sigma}(1)=1$, the identity bimultiplication. We note that the quotient algebra $A / C_{A}$ is isomorphic to the algebra of inner bimultiplications of $A$, which is the kernel of the natural homomorphism $\zeta: M_{A} \rightarrow P_{A}$. We identify them and define two maps

$$
\begin{aligned}
& \bar{\chi}_{1}: \Lambda \times \Lambda \rightarrow A / C_{A}, \\
& \bar{\chi}_{2}: N_{0} \rightarrow A / C_{A},
\end{aligned}
$$

such that

$$
\begin{aligned}
\bar{\chi}_{1}\left(\lambda_{1}, \lambda_{2}\right) & =\sigma\left(\lambda_{1} \lambda_{2}\right)-\sigma\left(\lambda_{1}\right) \sigma\left(\lambda_{2}\right), \\
\bar{\chi}_{2}\left(n_{0}\right) & =\sum_{1} k_{i} \sigma\left(\lambda_{i}\right),
\end{aligned}
$$

where $\lambda_{1}, \lambda_{2}, \lambda_{i} \in \Lambda, n_{0}=\sum_{i} k_{i}\left(\lambda_{i}\right), \quad \sum_{i} k_{i} \lambda_{i}=0$. Now $A$ and $A / C_{A}$ are $M_{A}$-bimodules and the natural map $\mu: A \rightarrow A / C_{A}$ which associates with every element of $A$ the inner bimultiplication of $A$ induced by it, is a homomorphism of $M_{A}$-bimodules. Thanks to the homomorphism $\sigma: \Gamma \rightarrow M_{A}$ we can consider $A$ and $A / C_{A}$ as $\Gamma$-bimodules and $\mu$ as a homomorphism of $\Gamma$-bimodules. We can now define two maps

$$
\begin{aligned}
& \chi_{1}: \Lambda \times \Lambda \rightarrow A \\
& \chi_{2}: N_{0} \rightarrow A
\end{aligned}
$$

such that $\mu \chi_{1}=\bar{\chi}_{1}, \mu \chi_{2}=\bar{\chi}_{2}$. It is easy to verify that the maps $\chi_{1}$ and $\chi_{2}$ satisfy all the six relations which the two maps $\chi_{1}$ and $\chi_{2}$, which determine a relative 2 -cochain, satisfy although here $A$ is not a $\Lambda$-bimodule. We now define four maps

$$
\begin{aligned}
& \pi_{1}: \Lambda \times \Lambda \times \Lambda \rightarrow C_{A}, \\
& \pi_{2}: \Lambda \times N_{0} \rightarrow C_{A}, \\
& \pi_{3}: N_{0} \times \Lambda \rightarrow C_{A}, \\
& \pi_{4}: N_{1} \rightarrow C_{A},
\end{aligned}
$$


exactly as in $\left[3\right.$, p. 200] in terms of $\chi_{1}$ and $\chi_{2}$. It is easy to verify that these four maps satisfy all the relations necessary to determine a relative 3-cochain with coefficients in the $\Lambda$-bimodule $C_{A}$. It can be shown as in [3, Theorem 5] that this relative 3 -cochain is a relative 3 -cocycle and that it determines an element of $H^{3}\left(\Lambda, \Gamma, C_{A}\right)$ which is independent of the choice of the map $\bar{\sigma}: \Lambda \rightarrow M_{A}$. This element is called the obstruction of $(\theta, \sigma)$. We are now in a position to answer the question raised at the beginning of this section.

THEOREM 2. The pair of regular homomorphisms $(\theta, \sigma)$ is induced by a relative extension if and only if the obstruction $\xi_{(\theta, \sigma)}=0$.

The proof is similar to that of [3, Theorem 6].

Finally, the $K$-module $H^{3}(\Lambda, \Gamma, M)$ can be interpreted in terms of the pairs of regular homomorphisms $(\theta, \sigma)$.

Theorem 3. Let $(\Lambda, \Gamma)$ be an algebra-couple and let $M$ be a $\Lambda$-bimodule. Let $f$ be a relative 3 -cocycle of $(\Lambda, \Gamma)$ with coefficients in $M$. Then there exist an algebra $A$ having $M$ as its bicentre and two regular homomorphisms $\theta: \Lambda \rightarrow P_{A}$ and $\sigma: \Gamma \rightarrow M_{A}$ satisfying the conditions (i), (ii), and (iii) described above such that $\theta$ induces over $M$ the given $\Lambda$ bimodule structure and that $f$ is the obstruction of the pair $(\theta, \sigma)$.

The proof is similar to that of [3, Theorem 8$]$, but one has to start with the algebra

$$
L=U_{0}+U_{0} \otimes_{V} U_{0}+\cdots+U_{0} \otimes_{V} \cdots \otimes_{V} U_{0}+\cdots
$$

instead of the algebra $L$ in [3, p. 205], in which the tensor products were taken over $K$.

REMARK. It would be interesting to investigate whether the relative cohomology proposed in this paper reduces to the absolute cohomology given in [3] when we take for $\Gamma$ the image of $K$ in $\Lambda$ under the natural homomorphism $K \rightarrow \Lambda$ which maps the identity of $K$ into the identity of $\Lambda$. If we denote the image of $K$ in $\Lambda$ by $K$ itself, it is evident that an absolute cochain of $\Lambda[3$, p. 182] is not a relative cochain of the algebra-couple $(\Lambda, K)$, since the maps which determirfe it need not satisfy any relations of the type described in $\$ 4$. Yet the interpretations of $H^{0}(\Lambda, \Gamma, M)$ and $H^{1}(\Lambda, \Gamma, M)$ show that $H^{0}(\Lambda, K, M)$ $\approx H^{0}(\Lambda, M)$ and $H^{1}(\Lambda, K, M) \approx H^{1}(\Lambda, M)$. Since the relative cohomology has been described in terms of the canonically constructed algebra-resolutions $U$ and $V$ of $\Lambda$ and $\Gamma$ respectively, the first task, as already indicated in the Introduction, is to construct a category of objects $(U, V, \Lambda, \Gamma, \epsilon)$ (cf. [3, Chapter II]) and to prove theorems analogous to $[3$, Theorems 1,2$]$. The second task will be to see 
whether we can take $V=\Gamma$, when $\Gamma$ is $K$-projective. If this is found possible, we shall have shown that the relative cohomology does reduce to the absolute cohomology when we take $\Gamma=K$.

\section{REFERENCES}

1. H. Cartan and S. Eilenberg, Homological algebra, Princeton Univ. Press, Princeton, N. J., 1956.

2. G. Hochschild, Relative homological algebra, Trans. Amer. Math. Soc. 82 (1956), 246-269.

3. U. Shukla, Cohomologie des algèbres associatives, Ann. Sci. Ecole Norm. Sup. 78 (1961), 163-209.

Kurukshetra University, Kurukshetra (Punjab), India

\section{DIRECT PROOF OF THE BASIC THEOREM ON MULTIPARTITE PARTITIONS}

E. M. WRIGHT ${ }^{1}$

In what follows all small latin letters denote non-negative integers, while $N$ and $N_{m s}$ are $j$-partite numbers, i.e. vectors or row-matrices of $j$ dimensions whose components are non-negative integers. In particular, $N=\left(n_{1}, n_{2}, \cdots, n_{j}\right)$. We write $q_{k}(N)$ for the number of partitions of $N$ into just $k$ parts and $r_{k}(N)$ for the number of partitions of $N$ into just $k$ different parts.

Let $\pi=\pi(k)$ be a partition of $k$ into $h(1)$ parts $1, h(2)$ parts 2 and so on, so that $k=\sum_{m} m h(m)$. We write

$$
H(\pi)=\prod_{m}\left\{h(m) ! m^{h(m)}\right\}^{-1}
$$

where, as usual, $0 !=1$, and $D(\pi, N)$ for the number of solutions of

$$
N=\sum_{m} \sum_{s=1}^{h(m)} m N_{m s},
$$

where the order of the $N_{m s}$ is relevant. Clearly

$$
D(\pi, N)=\prod_{i=1}^{j} D\left(\pi, n_{i}\right)
$$

Again, for $|X|<1$,

Received by the editors February 3, 1963.

1 Research sponsored by the Office, Chief of Research and Development, U. S. Department of the Army, through its European Research Office under contract DA91-591-EUC-2670. 